\title{
Synthesis of High Magnetic Moment CoFe Nanoparticles via Interfacial Diffusion in Core/Shell Structured Co/Fe Nanoparticles
}

\author{
Chao Wang ${ }^{1,2, \uparrow}$, Sheng Peng ${ }^{1, \dagger}$, Lise-Marie Lacroix ${ }^{1}$, Shouheng Sun $^{1}(\bowtie)$ \\ ${ }^{1}$ Department of Chemistry, Brown University, Providence, Rhode Island 02912, USA \\ ${ }^{2}$ Current Address: Materials Science Division, Argonne National Laboratory, Argonne, IL 60439, USA \\ Received: 25 December 2008 / Revised: 15 February 2009 / Accepted: 3 March 2009 \\ (C)Tsinghua University Press and Springer-Verlag 2009. This article is published with open access at Springerlink.com
}

\begin{abstract}
We report the synthesis of high magnetic moment CoFe nanoparticles via the diffusion of Co and Fe in core/ shell structured $\mathrm{Co} / \mathrm{Fe}$ nanoparticles. In an organic solution, Co nanoparticles were coated with a layer of Fe to form a $\mathrm{Co} / \mathrm{Fe}$ core/shell structure. Further raising the solution temperature led to inter-diffusion of $\mathrm{Co}$ and $\mathrm{Fe}$ and formation of CoFe alloy nanoparticles. These nanoparticles have high saturation magnetization of up to 192 $\mathrm{emu} / \mathrm{g} \mathrm{CoFe}$ and can be further stabilized by thermal annealing at $600{ }^{\circ} \mathrm{C}$.
\end{abstract}

\section{KEYWORDS}

CoFe nanoparticles, core/shell nanoparticles, magnetic nanoparticles, chemical synthesis

\section{Introduction}

High magnetic moment nanoparticles (NPs) are important for magnetic energy storage and magnetic data storage applications [1-4]. They are also superior contrast agents for highly sensitive magnetic resonance imaging (MRI) [5-10]. Body centered cubic (bcc) CoFe alloys have the highest saturation magnetization $\left(M_{\mathrm{s}}=\sim 240 \mathrm{emu} / \mathrm{g}\right)$ in this class of materials and are ideal magnetic materials for these applications. However, the chemical instability of nanostructured CoFe has made the synthesis of robust high magnetic moment CoFe NPs extremely challenging. Recent advances in NP syntheses have indicated that solution phase chemical synthesis is a reliable process for producing various monodisperse magnetic NPs by either simultaneous decomposition of $\mathrm{Co}\left(\eta^{3}-\mathrm{C}_{8} \mathrm{H}_{13}\right)\left(\eta^{4}-\mathrm{C}_{8} \mathrm{H}_{12}\right)\left(\right.$ or $\left.\mathrm{Co}\left(\mathrm{N}\left(\mathrm{SiMe}_{3}\right)_{2}\right)_{2}\right)$ and $\mathrm{Fe}(\mathrm{CO})_{5}[11]$, or co-reduction of $\mathrm{Co}(\mathrm{acac})_{2}$ and $\mathrm{Fe}(\mathrm{acac})_{3}(\mathrm{acac}=$ acetylacetonate) [12]. However, CoFe NPs made by these processes are chemically unstable, being subject to fast oxidation into various oxides unless a chemically inert carbonaceous shell is coated under high-temperature reaction conditions [7]. It has been demonstrated that interfacial diffusion between the core and the shell in core/ shell structured particles is a successful approach to various alloy NPs. For example, ferromagnetic FePt NPs were made by high-temperature reductive annealing of $\mathrm{FePt} / \mathrm{Fe}_{3} \mathrm{O}_{4} \mathrm{NPs}$ [13], and AuAg alloy NPs were synthesized from Ag/Au core/shell NPs [14]. Therefore, starting from monodisperse Co NPs, one may be able to make core/shell structured $\mathrm{Co} /$ Fe NPs and convert these to CoFe NPs with better

Address correspondence to ssun@brown.edu

${ }^{\dagger}$ These two authors made an equal contribution to the work. 
control over size, stability, and magnetic properties.

Here we report the synthesis of CoFe NPs via interfacial diffusion between $\mathrm{Co}$ and $\mathrm{Fe}$ in core/shell structured Co/Fe NPs. Monodisperse Co NP seeds were first synthesized by thermal decomposition of cobalt carbonyl, $\mathrm{Co}_{2}(\mathrm{CO})_{8}$, and were then coated with Fe by thermal decomposition of iron pentacarbonyl, $\mathrm{Fe}(\mathrm{CO})_{5}$. CoFe alloy NPs were formed by heating the core/shell structured $\mathrm{Co} / \mathrm{Fe} \mathrm{NPs}$ at $250{ }^{\circ} \mathrm{C}$ under an Ar atmosphere. The as-synthesized CoFe alloy NPs had the bcc structure and saturation magnetization of $192 \mathrm{emu} / \mathrm{g}$ CoFe. After thermal annealing at $600{ }^{\circ} \mathrm{C}$ in $\mathrm{Ar}$, the saturation magnetization of the NPs reached $230 \mathrm{emu} / \mathrm{g}$, close to the bulk value $(245 \mathrm{emu} / \mathrm{g}$ ) of CoFe alloy. More importantly, when exposed to the ambient environment for $24 \mathrm{~h}$, these CoFe NPs showed no obvious drop in saturation magnetization, indicating that the high magnetic moment alloy NPs are stable against oxidation and are suitable as building blocks for various high moment magnetic applications.

\section{Experimental}

All chemicals were used as received. $\mathrm{Co}_{2}(\mathrm{CO})_{8}$ and $\mathrm{Fe}(\mathrm{CO})_{5}$ were purchased from Strem Chemicals. Oleylamine $(70 \%)$, dioctylamine $(98 \%)$, oleic acid (90\%), 1,2,3,4-tetrahydronaphthalene (tetralin, 99\%), and octadecene (ODE, 90\%) were from SigmaAldrich.

Synthesis of Co NPs. In a typical synthesis of $8 \mathrm{~nm}$ Co NPs, $0.3 \mathrm{~mL}$ of oleic acid, and $0.5 \mathrm{~mL}$ of dioctylamine were mixed and magnetically stirred in 20 $\mathrm{mL}$ of tetralin. The solution was heated at $100{ }^{\circ} \mathrm{C}$ for $1 \mathrm{~h}$ under a nitrogen flow to remove traces of moisture. After cooling down to room temperature, $0.54 \mathrm{~g}$ of $\mathrm{Co}_{2}(\mathrm{CO})_{8}$ was quickly added into the solution. The solution was then heated up to $100^{\circ}$ $\mathrm{C}$ for $30 \mathrm{~min}$ and subsequently to $210{ }^{\circ} \mathrm{C}$ for $20 \mathrm{~min}$. After cooling down to room temperature, the Co NPs were precipitated by adding $20 \mathrm{~mL}$ of ethanol followed by centrifugation. The product was then dispersed in hexane and protected by argon.

Synthesis of CoFe alloy NPs. In a typical experiment, $20 \mathrm{~mL}$ of octadecene and $2 \mathrm{~mL}$ of oleylamine were mixed and magnetically stirred at

room temperature and then heated to $120{ }^{\circ} \mathrm{C}$ under an Ar atmosphere. After $30 \mathrm{~min}$ and under an Ar blanket atmosphere, $1 \mathrm{mmol}$ of $\mathrm{Fe}(\mathrm{CO})_{5}$ was added to the solution followed by $59 \mathrm{mg}$ of Co NP seeds in $2 \mathrm{~mL}$ of degassed hexane. The solution was kept at $120{ }^{\circ} \mathrm{C}$ for another $30 \mathrm{~min}$, and then slowly $\left(2-3^{\circ} \mathrm{C} / \mathrm{min}\right)$ heated to $180^{\circ} \mathrm{C}$ and kept at this temperature for $30 \mathrm{~min}$. The solution was further heated to $250^{\circ} \mathrm{C}$ for $15 \mathrm{~min}$. After cooling down to room temperature, the CoFe NPs were precipitated by adding $30 \mathrm{~mL}$ of isopropanol followed by centrifugation. The product was quickly dispersed in hexane and sealed under nitrogen for further applications.

In order to investigate the growth process, 0.2 $\mathrm{mL}$ aliquots of reaction solution were taken out by syringe after heating for $30 \mathrm{~min}$ at two different temperatures $\left(120^{\circ} \mathrm{C}\right.$ and $\left.180{ }^{\circ} \mathrm{C}\right)$, and quickly added to an excess of isopropanol to quench the reaction, and the resulting NPs were collected by centrifugation.

NP characterization. Samples for transmission electron microscopy (TEM) analysis were prepared by drying a dispersion of NPs on amorphous carbon-coated copper grids (Ted Pella). Particles were imaged using a Philips EM 420 transmission electron microscope (with an accelerating voltage of $120 \mathrm{kV}$ ). High-resolution TEM (HRTEM) images were recorded using a JEOL JEM-2010 microscope (with an accelerating voltage of $200 \mathrm{kV}$ ). X-ray diffraction (XRD) patterns of the particle assemblies were collected on a Bruker AXS D8 ADVANCE diffractometer with $\mathrm{Cu} K \alpha$ radiation $(\lambda=1.5418 \AA$ ) . Composition of alloy NPs was analyzed on a LEO 1560 scanning electron microscope (SEM) equipped with a spatially resolved energy dispersive X-ray spectroscopy (EDS) attachment. Magnetic studies were carried out using a Lakeshore 7404 highsensitivity vibrating sample magnetometer (VSM) with magnetic field up to $1.5 \mathrm{~T}$ at room temperature. The wt\% of metals in the NPs was obtained by measuring the weight difference before and after annealing the particles in a thermogravimetric analysis (TGA) pan at $800{ }^{\circ} \mathrm{C}$ under Ar for $1 \mathrm{~h}$.

\section{Results and discussion}

\section{黛 Springer}


Despite several recent reports [7, 11, 12], controlled alloying of $\mathrm{Co}$ and $\mathrm{Fe}$ in a single NP with tunable Co and Fe composition is still a challenging goal in synthesis. It is difficult to find $\mathrm{Co}$ and Fe precursors with chemical properties that have similar thermodynamics and growth kinetics under the same growth conditions. The rates of nucleation for $\mathrm{Co}$ and Fe are usually different, leading to limited control over the NP size and $\mathrm{Co}$ and Fe composition [15].

Previously, we have developed a synthetic scheme for AuAg alloy NPs involving annealing core/shell structured Ag/Au NPs [14]. Our further study indicated that such an interfacial elemental diffusion scheme could be readily applied to the synthesis of CoFe alloy NPs. This synthetic scheme is outlined in Fig. 1. In the synthesis, Co NP seeds are first coated with Fe by thermal decomposition of $\mathrm{Fe}(\mathrm{CO})_{5}$. Heating at a higher reaction temperature induces the elemental diffusion between $\mathrm{Co}$ and $\mathrm{Fe}$ at the core/ shell structure, forming CoFe alloy NPs.

Co NP seeds were synthesized by thermal decomposition of $\mathrm{Co}_{2}(\mathrm{CO})_{8}$ in the presence of oleic acid and dioctylamine. After a rapid washing process and proper protection under argon atmosphere, the fresh Co NPs were added to an octadecene solution containing oleylamine and $\mathrm{Fe}(\mathrm{CO})_{5}$ [8]. The Fe-coating on the Co NPs was achieved by thermal decomposition of $\mathrm{Fe}(\mathrm{CO})_{5}$ at $180{ }^{\circ} \mathrm{C}$. The $\mathrm{Co} / \mathrm{Fe}$ NPs were then annealed in the same solution at $250{ }^{\circ} \mathrm{C}$ to form CoFe alloy NPs.

Figure 2 shows TEM images of the Co NP seeds and CoFe NPs synthesized in this work. Figure 2(a) shows the $8 \mathrm{~nm}$ Co NPs with a size deviation of less than $5 \%$. The monodisperse Co NPs formed a hexagonal close-packed superlattice. Figures 2(b) and 2(c) are images of the CoFe NPs obtained by coating the Co seeds (Fig. 2(a)) followed by thermal annealing at $250{ }^{\circ} \mathrm{C}$. The NP size was increased from $8 \mathrm{~nm}$ to 11 $\mathrm{nm}$ due to addition of Fe. The atomic ratio of Co to Fe in the final product was measured to be 0.55 by EDS (Fig. S-1 in the Electronic Supplementary Material). Figure 2(d) shows the HRTEM image of a single CoFe NP. The inter-fringe distance was measured to

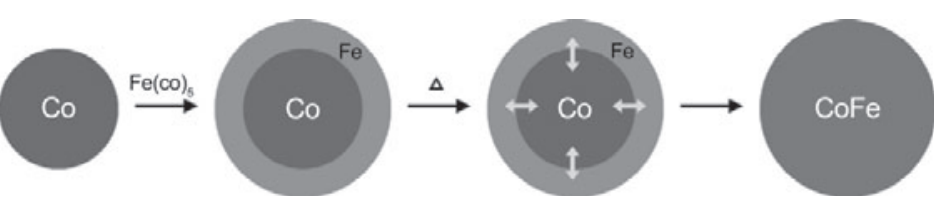

Figure 1 Schematic illustration of CoFe alloy NP synthesis by interfacial diffusion of $\mathrm{Co}$ and $\mathrm{Fe}$ in the core/shell structure Co/Fe NPs

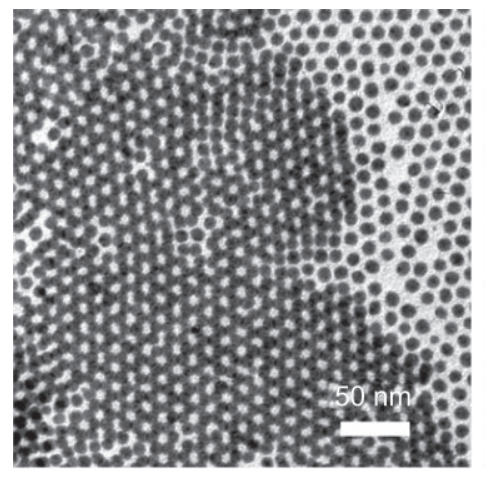

(a)

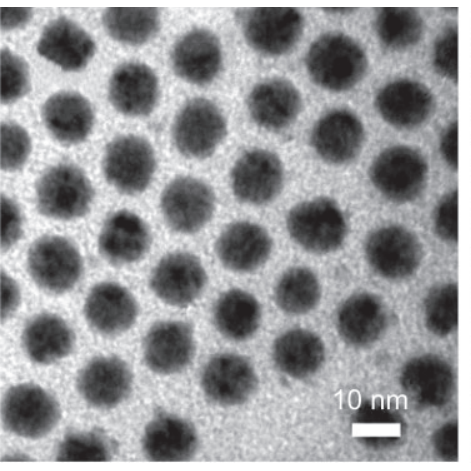

(c)

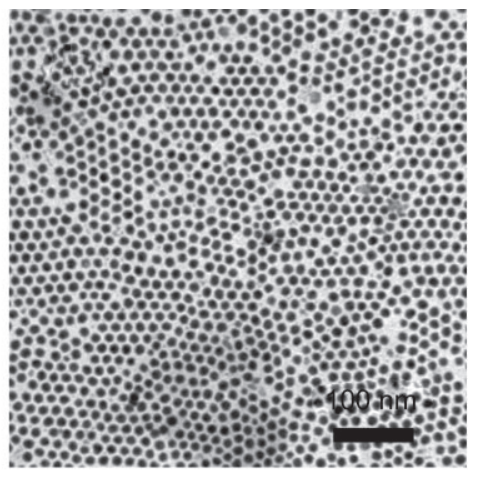

(b)

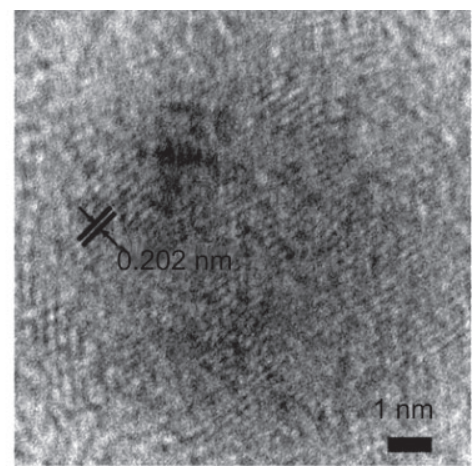

(d)
Figure 2 TEM images of (a) $8 \mathrm{~nm}$ Co seeds; (b) and (c) $11 \mathrm{~nm}$ CoFe NPs; (d) HRTEM image of a single CoFe NP

be $0.202 \mathrm{~nm}$, corresponding to (110) planes of the bcc phase of CoFe. The HRTEM image also shows that the NP had a polycrystalline structure.

In order to investigate the growth mechanism, the NPs were collected and analyzed at various stages of the growth. Figure 3 shows TEM images of the NPs obtained at $120^{\circ} \mathrm{C}$ and $180^{\circ} \mathrm{C}$. It can be seen that at $120{ }^{\circ} \mathrm{C}$, a 1-2 nm thick shell (gray) formed on some Co particles (dark). The gray shell is iron oxide coming from the facile oxidation of Fe when the NPs were exposed to air. The iron oxide shell has a much lower electron density than the metallic Co core and therefore looks much lighter. The TEM images also show some separate light species of 
irregular shapes with sizes of 5-10 nm in the sample collected at $120^{\circ} \mathrm{C}$. These are likely to be iron oxide NPs formed by separate Fe nucleation/growth in the reaction solution, implying incomplete consumption of $\mathrm{Fe}(\mathrm{CO})_{5}$ at this stage. However, the NPs collected at $180{ }^{\circ} \mathrm{C}$ had a much more uniform core/shell structure, with a shell thickness of $\sim 2 \mathrm{~nm}$. No free iron species can be seen at this stage. Upon further heating of the core/shell $\mathrm{Co} / \mathrm{Fe}$ at $250{ }^{\circ} \mathrm{C}$, the $\mathrm{Fe}$ (oxidized to iron oxide) shell is difficult to see in the TEM image and the NP size becomes $11 \mathrm{~nm}$ (Fig. 2), which indicates the diffusion of Fe into the Co core and the formation of CoFe alloy NPs. It should be noted that the diffusion of $\mathrm{Fe}$ into the Co core may start during the coating process. This diffusion may have a minimal effect on alloy formation due to the low coating temperatures and slow diffusion rate, however [14].

Figure 4 shows the XRD patterns of the assynthesized CoFe NPs and after annealing at $600{ }^{\circ} \mathrm{C}$ for $1 \mathrm{~h}$ (in Ar). The pattern after annealing corresponds to bcc CoFe. The peaks of the assynthesized NPs are very broad because of the polycrystalline nature of these NPs, as observed in the HRTEM image (Fig. 1(d)). The broad peak around $25^{\circ}$ is due to the presence of carbonaceous ligands on the particle surface. After annealing, the diffraction peaks become stronger and sharper, indicating enhanced crystallization of the NPs.

Magnetic properties of the CoFe NPs were studied by VSM. The as-synthesized NPs are superparamagnetic with saturation magnetization

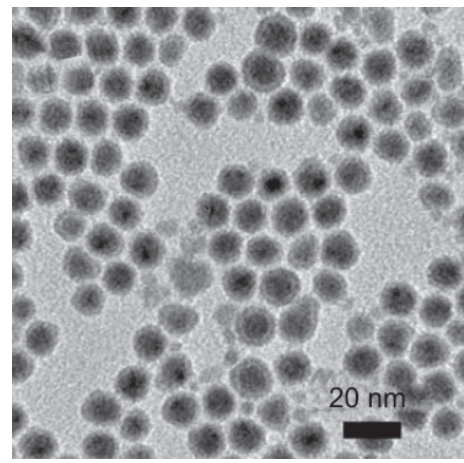

(a)

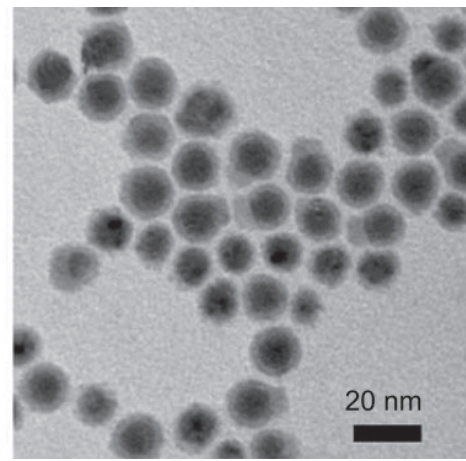

(b)

Figure 3 TEM images of the NPs collected at (a) $120^{\circ} \mathrm{C}$ and (b) $180^{\circ} \mathrm{C}$

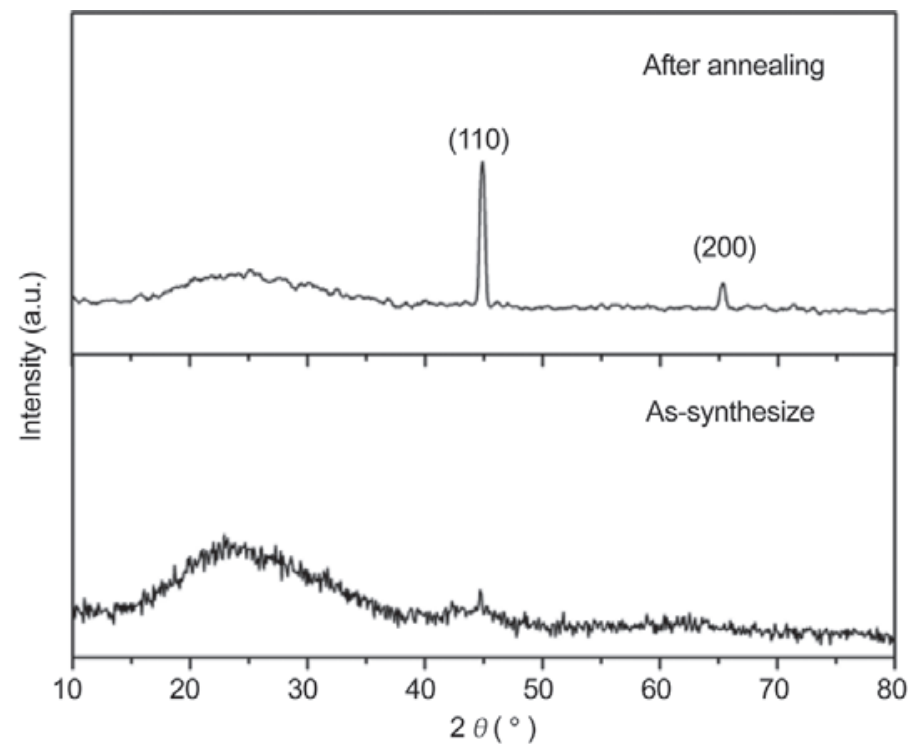

Figure 4 XRD patterns of as-synthesized CoFe NPs and after annealing at $600{ }^{\circ} \mathrm{C}$ for $1 \mathrm{~h}$ 
$\left(M_{\mathrm{s}}\right)$ up to $95 \mathrm{emu} / \mathrm{g}$ NPs. This corresponds to 192 emu/g CoFe metal (Fig. 5(a)), considering that 50.5\% by weight of the NPs was contributed by organic species (see Experimental section). The amount of organic species is rather high, due to the rapid washing process which leaves organic residues in the product. A saturation magnetization of $192 \mathrm{emu} / \mathrm{g}$ is $\sim 22 \%$ lower than the bulk value of CoFe alloy ( 245 $\mathrm{emu} / \mathrm{g}$ ) and the difference is likely caused by the polycrystalline nature of these NPs and by NP surface oxidation. After annealing in $\operatorname{Ar}\left(600{ }^{\circ} \mathrm{C}, 1 \mathrm{~h}\right)$ the saturation magnetization is increased to $230 \mathrm{emu} / \mathrm{g}$ $\mathrm{CoFe}$, probably owing to the increase in crystal grain size after annealing.

The as-synthesized NPs are not stable when exposed to air. Figure 5(b) shows the change of $M_{\mathrm{s}}$ for the as-synthesized CoFe NPs with air-exposure time. The value of $M_{\mathrm{s}}$ for the as-synthesized NPs (192 emu/g CoFe) was reduced to $\sim 140 \mathrm{emu} / \mathrm{g}$ after exposure for an hour due to the surface oxidation. At longer exposure times, the value of $M_{\mathrm{s}}$ reached a constant value of $\sim 120 \mathrm{emu} / \mathrm{g}$, suggesting that the formed thicker oxide layer resulted in much slow oxidation of the CoFe NPs. The chemical stability of the NPs was improved by high temperature thermal annealing. As indicated in Fig. 5(a), the annealed CoFe NPs showed a very small drop $(\sim 10 \mathrm{emu} / \mathrm{g}$ $\mathrm{CoFe}$ ) in magnetization after exposure to air for $24 \mathrm{~h}$. One possible reason for this enhanced chemical and

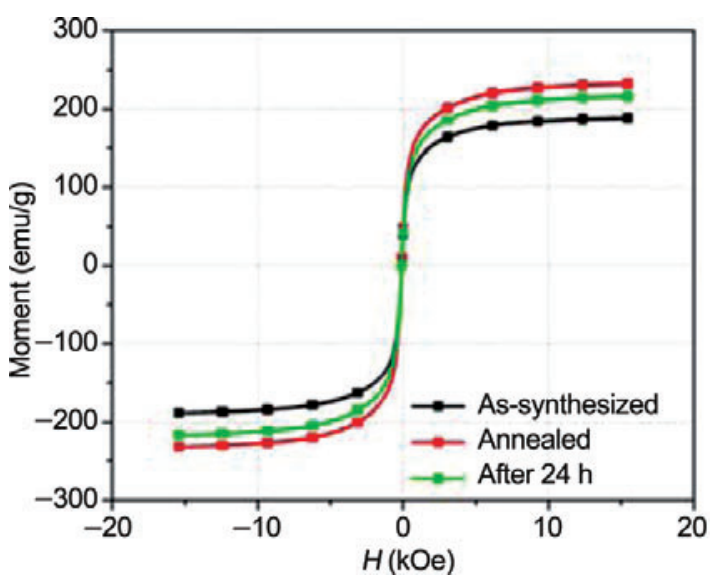

(a) magnetic stability is the formation of a carbonaceous coating from the organic surfactants around $\mathrm{CoFe}$ NPs during the high-temperature annealing process [7]. It can also be due to the crystallization of the surface oxides (formed when exposed to air) under annealing; a similar observation has been reported in the synthesis and stabilization of Fe NPs [8].

\section{Conclusions}

The paper reports a new synthesis of high magnetic moment CoFe NPs in an organic solution phase from core/shell structured Co/Fe NPs via the interfacial diffusion of $\mathrm{Co}$ and $\mathrm{Fe}$ in the core/shell structure at $250{ }^{\circ} \mathrm{C}$. The unique feature of this synthesis is that it uses monodisperse Co NPs as seeds and by controlling the Fe coating, monodisperse $\mathrm{Co} / \mathrm{Fe}$ NPs and therefore CoFo NPs can be readily produced. The as-synthesized CoFe NPs are polycrystalline with saturation magnetization reaching $192 \mathrm{emu} / \mathrm{g}$ CoFe metal and are not air-stable unless they are annealed at $600{ }^{\circ} \mathrm{C}$. These high magnetic moment NPs may serve as building blocks for fabricating nanocomposite magnets with maximum energy product or for other nanomagnetic applications.

\section{Acknowledgements}

This work was supported by NSF/DMR 0606264, ONR/MURI N00014-05-1-0497 and the Brown

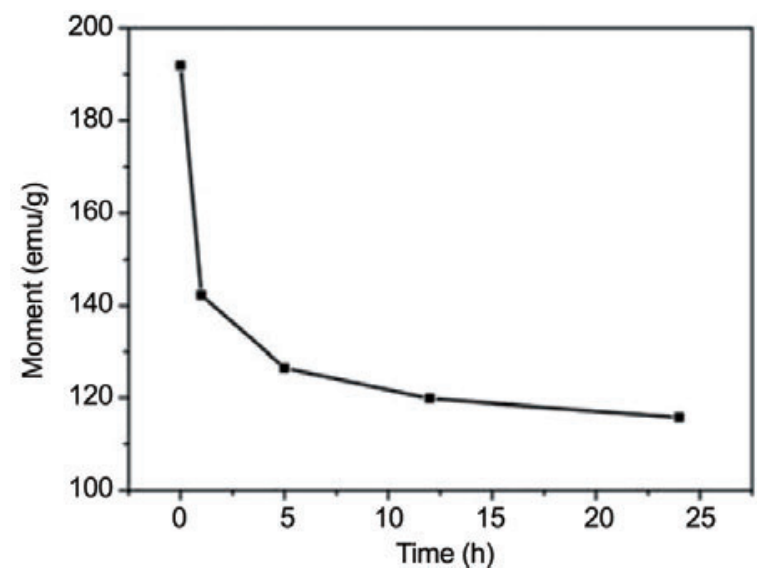

(b)

Figure 5 (a) Hysteresis loops of the as-synthesized (black) and the annealed (red) CoFe NPs, and the annealed (600 ${ }^{\circ} \mathrm{C}$ in Ar for $1 \mathrm{~h}) \mathrm{NPs}$ after $24 \mathrm{~h}$ of exposure in air (green); (b) dependence of saturation magnetization $\left(M_{s}, \mathrm{emu} / \mathrm{g}\right.$ CoFe) of the NPs on time exposed to air in hexane dispersion 
University Seed Fund. Lise-Marie Lacroix thanks the French State/D4S grant for supporting her work at Brown University.

Electronic Supplementary Material: The EDS spectrum of CoFe NPs is available in the online version of this article at http://dx.doi.org/10.1007/ s12274-009-9037-4 and is accessible free of charge.

\section{References}

[1] Sun, S.; Murray, C. B.; Weller, D.; Folks, L.; Moser, A. Monodisperse FePt nanoparticles and ferromagnetic FePt nanocrystal superlattices. Science 2000, 287, 1989-1992.

[2] Lacheisserie, E. T.; Gignoux, D.; Schlenker, M. Magnetism: Materials and Spplications; Springer, 2004.

[3] Bader, S. D. Colloquium: Opportunities in nanomagnetism. Rev. Mod. Phys. 2006, 78, 1-15.

[4] Lu, A.-H.; Salabas, E. L.; Schüth, F. Magnetic nanoparticles: Synthesis, protection, functionalization, and application. Angew. Chem. Int. Ed. 2007, 46, 12221244.

[5] Lewin, M.; Carlesso, N.; Tung, C. -H.; Tang, X. -W.; Cory, D.; Scadden, D. T.; Weissleder, R. Tat peptide-derivatized magnetic nanoparticles allow in vivo tracking and recovery of progenitor cells. Nat. Biotechnol. 2000, 22, 47-52.

[6] Zhao, M.; Deauregard, D. A.; Loizou, L.; Davletov, B.; Brindle, K. M. Non-invasive detection of apoptosis using magnetic resonance imaging and a targeted contrast agent. Nat. Med. 2001, 7, 1241-1244.

[7] Seo, W. S.; Lee, J. H.; Sun, X.; Suzuki, Y.; Mann, D.; Liu, Z.;
Terashima, M.; Yang, P. C.; Mcconnell, M. V.; Nishimura, D. G.; Dai, H. FeCo/graphitic-shell nanocrystals as advanced magnetic-resonance-imaging and near-infrared agents. Nat. Mater. 2006, 5, 971-976.

[8] Peng, S.; Wang, C.; Xie, J.; Sun, S. Synthesis and stabilization of monodisperse Fe nanoparticles. J. Am. Chem. Soc. 2006, 128, 10676-10677.

[9] Xu, C.; Xie, J.; Ho, D.; Wang, C.; Kohler, N.; Walsh, E. G.; Morgan, J. R.; Chin, Y. E.; Sun, S. $\mathrm{Au}-\mathrm{Fe}_{3} \mathrm{O}_{4}$ dumbbell nanoparticles as dual-functional Probes. Angew. Chem. Int. Ed. 2008, 47, 173-176.

[10] Sun, C.; Lee, J.; Zhang, M. Magnetic nanoparticles in MR imaging and drug delivery. Adv. Drug Deliv. Rev. 2008, $60,1252-1265$.

[11] Desvaux, C.; Amiens, C.; Fejes, P.; Renaud, P.; Respaud, M.; Lecante, P.; Snoeck, E.; Chaudret, B. Multimillimetrelarge superlattices of air-stable iron-cobalt nanoparticles. Nat. Mater. 2005, 4, 750-753.

[12] Chaubey, G. S.; Barcena, C.; Paudyal, N.; Rong, C.; Gao, J.; Sun, S.; Liu, J. P. Synthesis and stabilization of FeCo nanoparticles. J. Am. Chem. Soc. 2007, 129, 7214 7215.

[13] Kim, J.; Rong, C.; Lee, Y.; Liu, J. P.; Sun, S. From core/ shell structured FePt/FeO/MgO to ferromagnetic FePt nanoparticles. Chem. Mater. 2008, 20, 7242-7245.

[14] Wang, C.; Peng, S.; Chan, R.; Sun, S. Synthesis of AuAg alloy nanoparticles from core/shell structured $\mathrm{Ag} / \mathrm{Au}$. Small, in press, DOI:10.1002/smll.200801169.

[15] Hütten, A.; Sudfeld, D.; Ennen, I.; Reiss, G.; Wojczykowski, K.; Jutzi, P. Ferromagnetic FeCo nanoparticles for biotechnology. J. Mag. Mag. Mater. 2005, 293, 93-101. 